04

\title{
Двумерная модель разряда Пеннинга в цилиндрической камере с осевым магнитным полем
}

\author{
(c) С.T. Суржиков ${ }^{1,2}$ \\ ${ }^{1}$ Институт проблем механики им. А.Ю. Ишлинского РАН, \\ 119526 Москва, Россия \\ ${ }^{2}$ Всероссийский научно-исследовательский институт автоматики им. Н.Л. Духова, \\ 127055 Москва, Россия \\ e-mail: surg@ipmnet.ru
}

(Поступило в Редакцию 10 сентября 2016 г.)

\begin{abstract}
Представлена диффузионно-дрейфовая модель разряда Пеннинга в молекулярном водороде при давлениях порядка 1 Torr с учетом внешней электрической цепи. Рассмотрена двумерная осесимметричная геометрия разряда с цилиндрическим анодом и плоскими катодами, расположенными перпендикулярно оси симметрии. Внешнее магнитное поле с индукцией порядка $0.1 \mathrm{~T}$ приложено в осевом направлении. С использованием созданной диффузионно-дрейфовой модели выполнено численное моделирование электродинамической структуры пеннинговского разряда в диапазоне давлений 0.5-5 Torr, при ЭДС источника тока $200-500 \mathrm{~V}$. C использованием численных экспериментов изучена эволюция электродинамической структуры разряда при изменении давления без внешнего магнитного поля (режим классического тлеющего разряда) и в осевом магнитном поле (разряд Пеннинга). Расчетным путем подтверждены теоретические предсказания о существовании в пеннинговском разряде осредненного движения электронов и ионов не только в осевом и радиальном направлениях, но и в азимутальном направлении.
\end{abstract}

DOI: $10.21883 /$ JTF.2017.08.44722.2031

\section{Введение}

Особенностью разряда Пеннинга является определяющая роль магнитного поля, обеспечивающего ионизацию нейтрального газа при давлениях менее $p<1$ mTorr и напряженности электрического поля $E \sim 10^{3} \mathrm{~V} / \mathrm{cm}$ в газоразрядных устройствах, когда разряд самостоятельно без магнитного поля существовать не может.

Разряд Пеннинга был открыт около ста лет назад [1] и нашел широкое применение в практике фундаментальных и прикладных исследований [2]. Среди наиболее эффективных его применений - ускоритель ионов водорода и дейтерия в газонаполненных нейтронных трубках [3] а также как источник ионов в ускорителях заряженных частиц [4].

Типичная геометрия разряда Пеннинга показана на рис. 1. Сплошной анод, в форме полого цилиндра, ограничен с торцов (с некоторым зазором или изолятором) дисковыми секциями катода, в одной из которых имеется отверстие в приосевой области, откуда извлекаются ионы (как правило, водорода, дейтерия, ксенона, аргона), впоследствии ускоряемые до необходимой энергии. Типичные давления в газоразрядной камере порядка 1 mTorr, так что в отсутствие магнитного поля длина свободного пробега электронов превышает типичные размеры камеры и эффективность ионизации слишком мала для поддержания разряда. Качественно физика процессов в такой разрядной камере анализировалось в книге [2], а также в ряде оригинальных и расчетных работ [2,5-10]. Однако до настоящего времени в литературе отсутствуют расчетно-теоретические рабо- ты, в которых исследована пространственная структура пеннинговского разряда. В настоящей работе для схемы рис. 1 изучается электродинамика стационарного разряда Пеннинга в молекулярном водороде при относительно высоких давлениях $p \sim 1$ Torr, напряженности электрического поля $E \sim 200-600 \mathrm{~V} / \mathrm{cm}$ и падениях напряжения между электродами $V \sim 300 \mathrm{~V}$. В этих условиях длина свободного пробега электронов $l \sim 0.02 \mathrm{~cm}$, поэтому является допустимым использование классической формулы Таунсенда для ионизации молекул электронными ударом и диффузионно-дрейфовой модели тлеющего разряда [11]. Заметим, что использование указанной физически обоснованной модели является также некоторой дополнительной гарантией получения физически достоверных результатов в условиях отсутствия соответствующих экспериментальных данных.

Учитывая, что тлеющий разряд представляет собой частично ионизованный газ с типичной концентрацией заряженных частиц $\sim 10^{11} \mathrm{~cm}^{-3}$ на фоне $\sim 10^{17} \mathrm{~cm}^{-3}$ нейтральных частиц и малой абсолютной величиной токов через разряд (десятки миллиампер), представляется очевидным, что внешнее магнитное поле $B \sim 0.01-1 \mathrm{~T}$ может оказать сильное влияние на структуру тлеющего разряда. При этом указанное магнитное поле не будет искажаться самим разрядом. Данное допущение положено в основу данной численной модели тлеющего разряда во внешнем магнитном поле.

Целью настоящего исследования является изучение электродинамической структуры пеннинговского разряда в диапазоне давлений $p=0.5-5$ Torr и анализ закономерностей изменения скоростей ионов, падающих на 


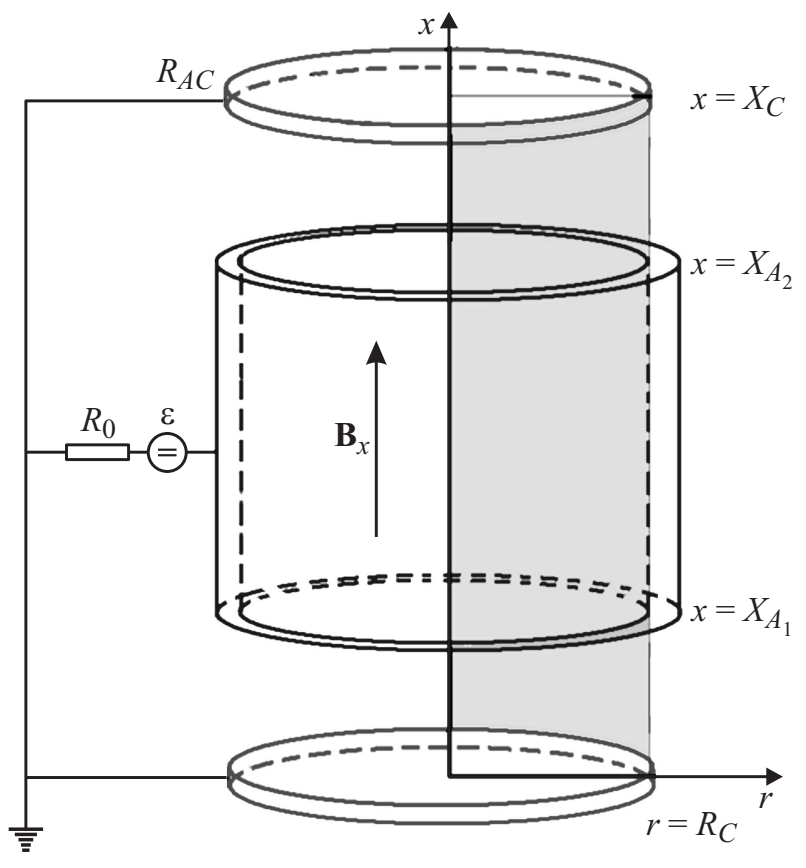

Рис. 1. Схема разряда Пеннинга с внешним магнитным полем.

катод при вариации таких параметров разряда, как давления, ЭДС, индукции магнитного поля и высоты анода. Первые результаты этого исследования приведены в [12].

\section{Уравнение диффузионно-дрейфовой модели разряда Пеннинга}

Уравнения диффузионно-дрейфовой модели тлеющего разряда в двумерной цилиндрической геометрии с продольным (осевым) магнитным полем были сформулированы в работе [13] при изучении структуры столба нормального тлеющего разряда между „бесконечными“ плоскими электродами. Указанная система уравнений используется в данной работе с соответствующими решаемой задаче граничными условиями, что позволяет моделировать пеннинговский разряд:

$$
\begin{gathered}
\frac{\partial n_{e}}{\partial t}+\frac{\partial}{\partial x}\left(-\mu_{e} n_{e} E_{x}-D_{e} \frac{\partial n_{e}}{\partial x}\right)+\frac{1}{r} \frac{\partial}{\partial r} r\left(-\frac{\mu_{e}}{1+b_{e}^{2}} n_{e} E_{r}\right. \\
\left.-\frac{D_{e}}{1+b_{e}^{2}} \frac{\partial n_{e}}{\partial y}\right)=\alpha\left|\Gamma_{e}\right|-\beta n_{e} n_{i}=\omega, \\
\frac{\partial n_{i}}{\partial t}+\frac{\partial}{\partial x}\left(\mu_{i} n_{i} E_{x}-\frac{D_{i}}{1+b_{i}^{2}} \frac{\partial n_{i}}{\partial x}\right)+\frac{1}{r} \frac{\partial}{\partial r} r\left(\frac{\mu_{i}}{1+b_{i}^{2}} n_{i} E_{r}\right. \\
\left.-\frac{D_{i}}{1+b_{i}^{2}} \frac{\partial n_{i}}{\partial y}\right)=\alpha\left|\Gamma_{e}\right|-\beta n_{e} n_{i}=\omega, \\
\frac{\partial^{2} \varphi}{\partial x^{2}}+\frac{1}{r} \frac{\partial}{\partial r} r \frac{\partial \varphi}{\partial r}=4 \pi e\left(n_{e}-n_{i}\right),
\end{gathered}
$$

где $n_{e}, n_{e}$ - числовые концентрации электронов и ионов; $x, r$ - осевая и радиальная координаты; $\mu_{e}=e / m_{e} v_{e}, \mu_{i}=e / m_{i} v_{i n}-$ подвижности электронов и ионов; $m_{e}, m_{i}$ - масса электрона и иона; $v_{e n}, v_{e i}, v_{i n}$ частоты столкновений электронов с нейтральными частицами, с ионами, а также ионов с нейтралами; $v_{e}=v_{e n}+v_{e i} ; D_{e}=\left(k T_{e} / e\right) \mu_{e}, D_{i}=\left(k T_{i} / e\right) \mu_{i}$ - коэффициенты диффузии электронов и ионов; $T_{e}, T_{i}$ - температура электронов и ионов: $E_{x}, E_{r}-$ компоненты электрического поля $\mathbf{E}=-\operatorname{grad} \varphi ; \varphi-$ электрический потенциал; $b_{e}=\mu_{e} B_{x} / c=\omega_{e} / \nu_{e}, b_{i}=\mu_{i} B_{x} / c=\omega_{i} / \nu_{i n}$ параметры Холла электронной и ионной жидкостей; $\omega_{e}=e B_{x} / m_{e} c, \omega_{i}=e B_{x} / m_{i} c-$ ларморовские частоты электронов и ионов; $B_{x}-x$-я компонента вектора индукции магнитного поля $\mathbf{B} ; k, e, c-$ постоянная Больцмана, заряд электрона и скорость света.

В правой части уравнений (1) и (2) содержатся источниковые слагаемые, отвечающие ионизации нейтрального газа при столкновениях с электронами и рекомбинации при электронно-ионных столкновениях; $\alpha, \beta$ - коэффициенты ионизации (первый коэффициент Таунсенда) и рекомбинации. Как видно из (1), (2), скорость ионизации пропорциональна плотности потока электронов, пронизывающих элементарный физический объем с координатами $(x, r)$, которая в рассматриваемом случае тлеющего разряда с продольным магнитным полем имеет три компоненты [13]:

$$
\begin{gathered}
\left|\Gamma_{e}\right|=\sqrt{\Gamma_{e, x}^{2}+\Gamma_{e, r}^{2}+\Gamma_{e, \varphi}^{2}}, \\
\Gamma_{e, x}=n_{e} v_{e, x}=-\mu_{e} n_{e} E_{x}-D_{e} \frac{\partial n_{e}}{\partial x}, \\
\Gamma_{e, r}=n_{e} v_{e, r}=-\frac{\mu_{e}}{1+b_{e}^{2}} n_{e} E_{r}-\frac{D_{e}}{1+b_{e}^{2}} \frac{\partial n_{e}}{\partial r}, \\
\Gamma_{e, \varphi}=n_{e} v_{e, \varphi}=-b_{e} \Gamma_{e, r},
\end{gathered}
$$

где $v_{e, x}, v_{e, r}, v_{e, \varphi}-$ проекции усредненной скорости движения электронов $\mathbf{u}_{e}$ на оси цилиндрической системы координат. Заметим, что компоненты плотности потока ионов в левой части (2)

$$
\begin{gathered}
\Gamma_{i, x}=n_{i} v_{i, x}=+\mu_{i} n_{i} E_{x}-D_{i} \frac{\partial n_{i}}{\partial x}, \\
\Gamma_{i, r}=n_{i} v_{i, r}=+\frac{\mu_{e}}{1+b_{i}^{2}} n_{i} E_{r}-\frac{D_{i}}{1+b_{i}^{2}} \frac{\partial n_{i}}{\partial r}
\end{gathered}
$$

играют важную роль в формировании пространственного распределения ионов в газоразрядном промежутке, а плотность потока ионов в азимутальном направлении

$$
\Gamma_{i, \varphi}=n_{i} v_{i, \varphi}=-b_{i} \Gamma_{i, r}
$$

определяет скорость усредненного вращения ионов вокруг оси симметрии. Здесь $v_{i, x}, v_{i, r}, v_{i, \varphi}-$ проекции усредненной скорости движения ионов $\mathbf{u}_{i}$ на оси цилиндрической системы координат. 


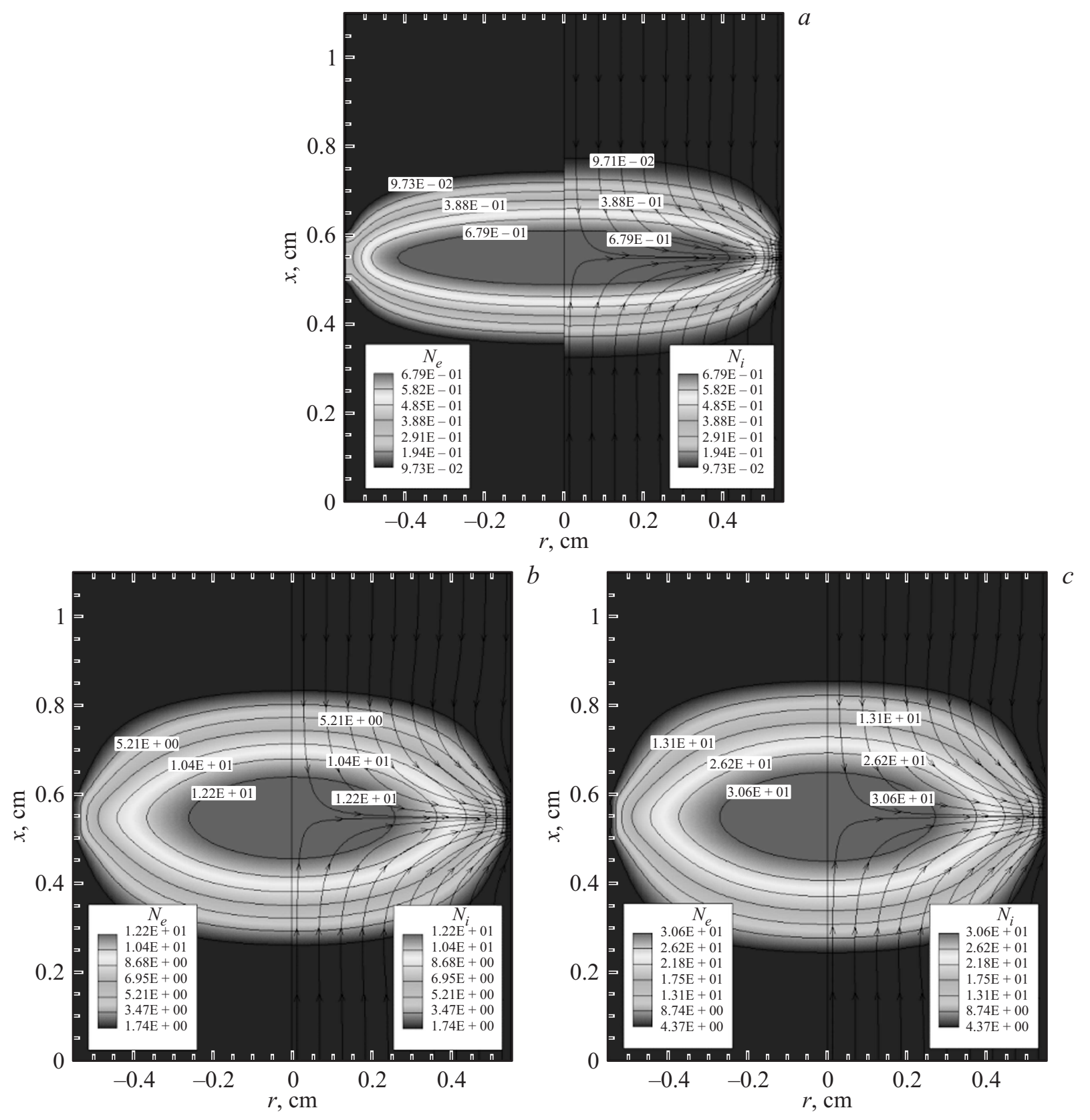

Рис. 2. Концентрация электронов (слева) и ионов (справа), $N_{e}, N_{i}, 10^{10} \mathrm{~cm}^{-3}$, в пеннинговском разряде с высотой анода $H_{A}=0.1 \mathrm{~cm}$ при $p=1$ Torr, $B_{x}=0.1 \mathrm{~T}, \varepsilon=100(a), 200(b), 300 \mathrm{~V}(c)$.

Заметим, что при выводе уравнений (1) и (2) использовались следующие допущения:

$$
m_{e} \ll m_{i}, \quad\left|\mathbf{u}_{e}\right| \gg\left|\mathbf{u}_{n}\right|, \quad m_{e} v_{e} \ll m_{i} v_{i n},
$$

где $\mathbf{u}_{n}-$ усредненная скорость движения нейтральных частиц.

Граничные условия для определения концентраций ионов и электронов, а также электрического потенциала формулируются в следующем виде:

$$
x=0, r \in\left[0, R_{e}\right]: \quad \frac{\partial n_{i}}{\partial x}=0, \Gamma_{e}=\gamma \Gamma_{i}, \varphi=0,
$$

$$
\begin{gathered}
x=X_{C}: r \in\left[0, R_{A C}\right]: \frac{\partial n_{i}}{\partial x}=0, \Gamma_{e}=\gamma \Gamma_{i}, \varphi=0, \\
r=0, \quad \frac{\partial n_{e}}{\partial r}=\frac{\partial n_{i}}{\partial r}=\frac{\partial \varphi}{\partial r}=0, \\
r=R, \quad x<X_{A_{1}}, \quad x>X_{A_{2}}: \frac{\partial n_{e}}{\partial r}=\frac{\partial n_{i}}{\partial r}=\frac{\partial \varphi}{\partial r}=0, \\
r=R, X_{A_{1}}<x<X_{A_{2}}: \frac{\partial n_{e}}{\partial r}=0, \quad n_{i}=0, \quad \varphi=V,
\end{gathered}
$$

где $\gamma$ - коэффициент вторичной ион-электронной эмиссии, $V$ - падение напряжения между анодной и катодными секциями. 
В качестве начальных условий в центре разрядной камеры задавалось плазменное облако с концентрацией частиц $n_{e}=n_{i}=10^{10} \mathrm{~cm}^{-3}$.

Использовались традиционные замыкающие соотношения [11]

$$
\begin{gathered}
\mu_{e}(p)=\frac{4.5 \cdot 10^{5}}{p} \mathrm{~cm}^{2} /(\mathrm{V} \cdot \mathrm{s}), \\
\mu_{i}(p)=\frac{6552.2}{p} \mathrm{~cm}^{2} /(\mathrm{V} \cdot \mathrm{s}), \\
D_{e}=\mu_{e}(p) T_{e} \mathrm{~cm}^{2} / \mathrm{s}, \quad D_{i}=\mu_{i}(p) T \mathrm{~cm}^{2} / \mathrm{s}
\end{gathered}
$$

где $p-$ давление в тлеющем разряде в Torr, $T=300 \mathrm{~K}$ - температура нейтральных частиц.
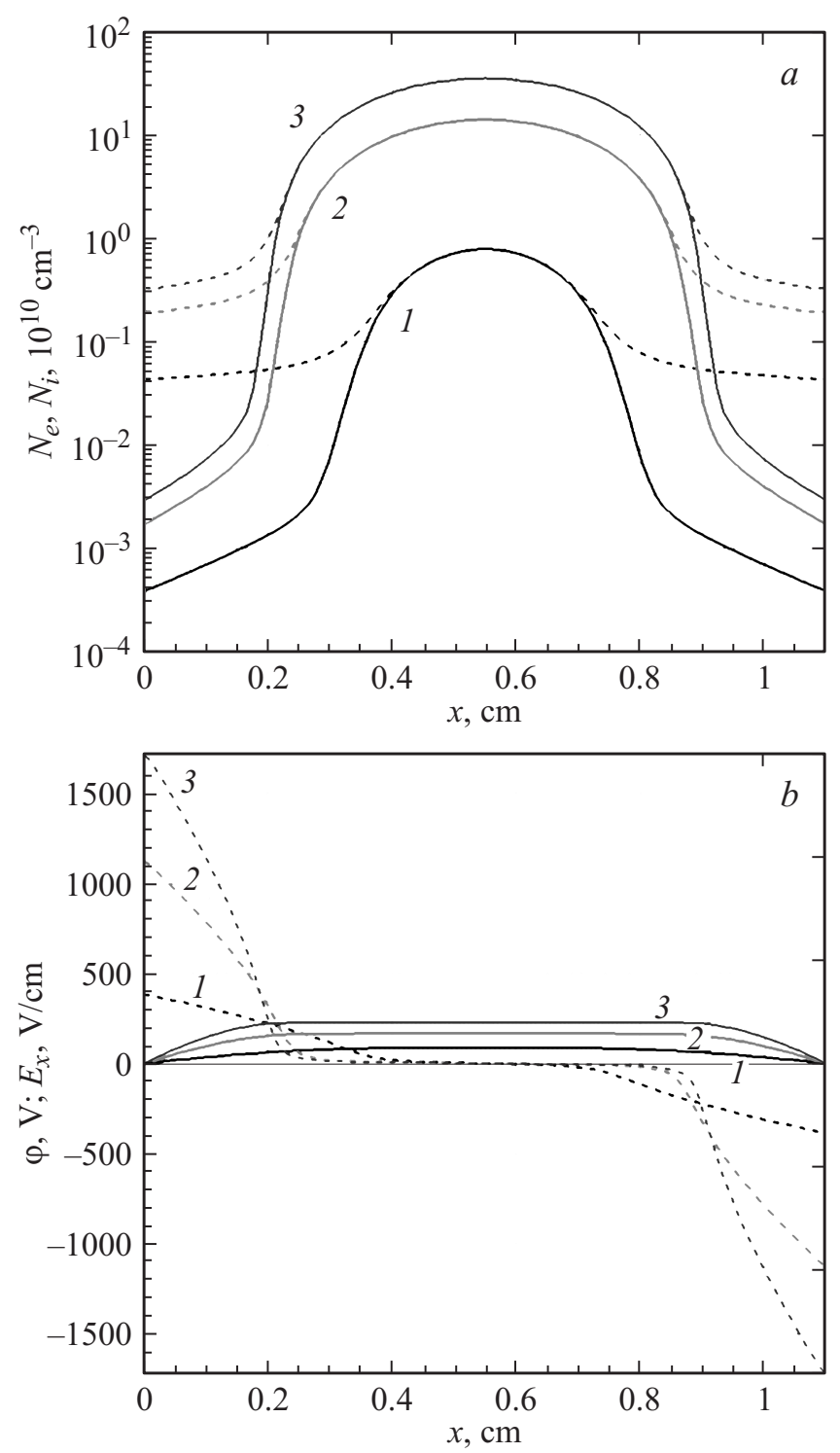

Рис. 3. Распределение концентраций электронов (сплошные кривые) и ионов (штриховые кривые) $(a)$, электрического потенциала (сплошные кривые) и напряженности электрического поля $E_{x}$ (штриховые кривые) $(b)$ вдоль оси симметрии пеннинговского разряда с анодом высотой $H_{A}=0.1 \mathrm{~cm}$ при $p=1$ Torr, $B_{x}=0.1 \mathrm{~T}, \varepsilon=100(1), 200(2), 300 \mathrm{~V}(3)$.
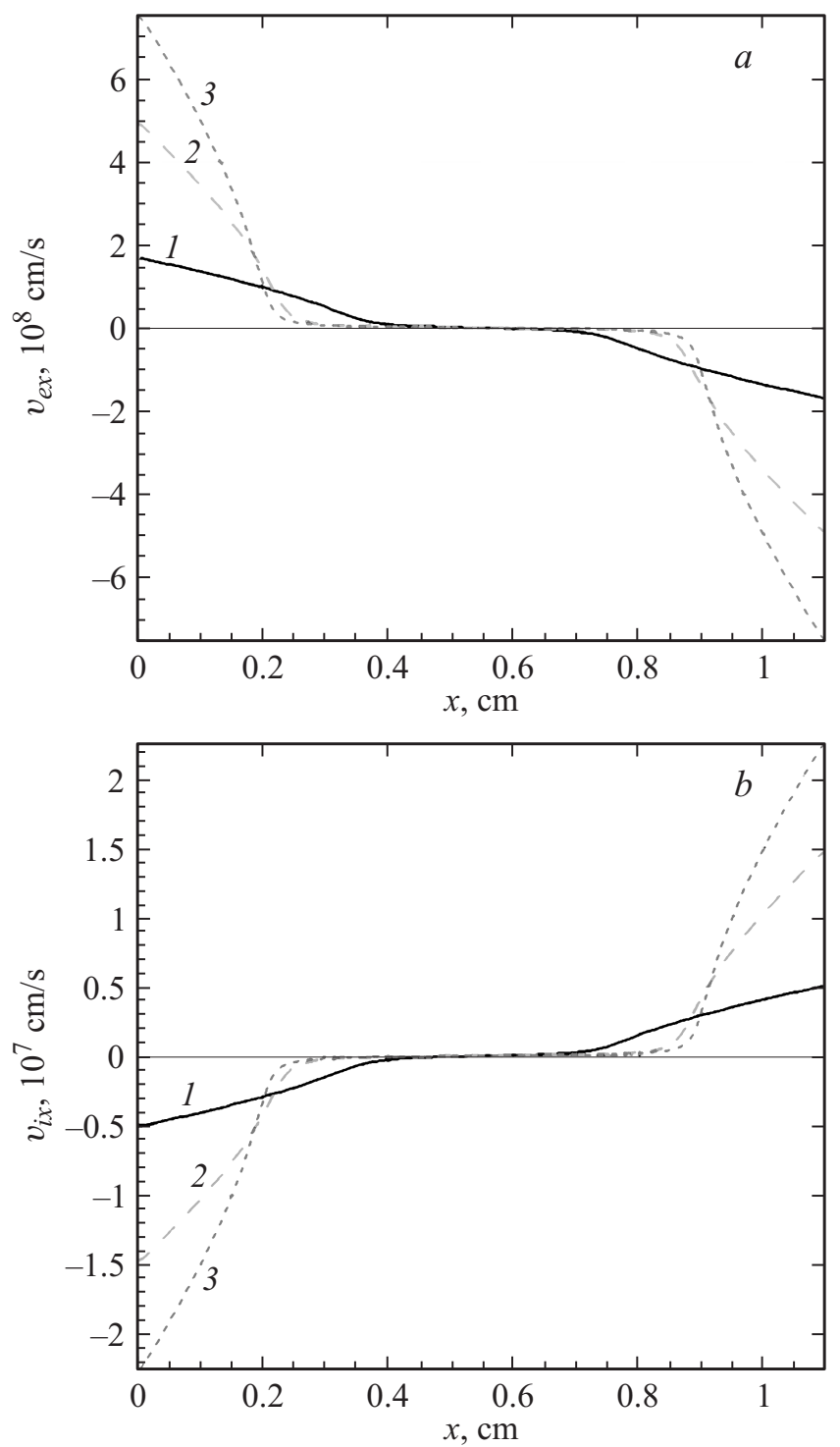

Рис. 4. Распределение осевой скорости электронов вдоль $v_{e, x}$ и ионов $v_{i, x}$ вдоль оси симметрии пеннинговского разряда с анодом высотой $H_{A}=0.1 \mathrm{~cm}$ при $p=1$ Torr, $B_{x}=0.1 \mathrm{~T}$, $\varepsilon=100$ (1), 200 (2), $300 \mathrm{~V}(3)$.

Коэффициент ион-электронной рекомбинации и температура электронов приняты постоянными:

$$
\beta=2 \cdot 10^{-7} \mathrm{~cm}^{3} / \mathrm{s}, \quad T_{e}=11610 \mathrm{~K},
$$

Коэффициент ионизации (1-й коэффициент Таунсенда) задавался в виде

$$
\alpha(E)=p A \exp \left[-\frac{B}{(|\mathbf{E}| / p)}\right] \mathrm{cm}^{-1},
$$

где аппроксимационные коэффициенты для молекулярного водорода

$$
A=5 \frac{1}{\mathrm{~cm} \text { Torr }}, \quad B=130 \frac{V}{\mathrm{~cm} \text { Torr }}
$$


в формуле Таунсенда (15) справедливы в диапазоне приведенных полей

$$
\frac{E}{p} \in[150,600] \mathrm{V} /(\mathrm{cm} \text { Torr }) .
$$

Уравнения диффузионно-дрейфовой модели решались совместно с уравнением внешней электрической цепи, которое для постоянного тока имеет вид

$$
\varepsilon=V+I R_{0}
$$

где $I$ - полный ток через разрядный промежуток, $\varepsilon-$ ЭДС источника питания, $R_{0}-$ сопротивление внешней цепи (рис. 1).

В рассматриваемой постановке полный ток через разряд может быть выполнен интегрированием плотности тока на аноде, катоде $(x=0)$ и антикатоде $\left(x=X_{C}\right)$ :

$$
\begin{gathered}
I_{A}=2 \pi R_{C} \int_{X_{A_{1}}}^{X_{A_{2}}} j_{A} d x, \quad j_{A}=e\left(\mu_{e} n_{e} \frac{\partial \varphi}{\partial r}\right)_{r=R_{C}, x \in\left[X_{A_{1}}, X_{A_{1}}\right]}, \\
I_{C}=2 \pi \int_{0}^{R_{C}} j_{C} r d r, j_{C}=e(1+\gamma)\left(\mu_{i} n_{i} \frac{\partial \varphi}{\partial r}\right)_{x=0, r \in\left[0, R_{C}\right]}, \\
I_{A C}=2 \pi \int_{0}^{R_{C}} j_{A C} r d r, j_{A C}=e(1+\gamma)\left(\mu_{i} n_{i} \frac{\partial \varphi}{\partial r}\right)_{x=X_{C}, r \in\left[0, R_{C}\right]} .
\end{gathered}
$$

Заметим, что в данной постановке (в стационарных условиях существования разряда) должны выполняться два очевидных условия

$$
I_{A}=I_{C}+I_{C A}, \quad I_{C}=I_{C A},
$$

которые использовались для контроля точности численного моделирования. Численный метод решения системы уравнений диффузионно-дрейфовой модели представлен в [14].

\section{Результаты численного моделирования}

Расчеты проводились для разряда Пеннинга в молекулярном водороде при следующих исходных данных: давление $p=0.1-5.0$ Torr, $\varepsilon=200-500 \mathrm{~V} ; R_{0}=3 \mathrm{k} \Omega$, $\gamma=0.33, \quad B_{x}=0.1-0.6 \mathrm{~T}, \quad R_{C}=R_{A C}=0.55 \mathrm{~cm}, \quad X_{C}=$ $=1.1 \mathrm{~cm}$. Рассматривались две высоты анода: $H_{A}=$ $=0.1 \mathrm{~cm} \quad\left(X_{A_{1}}=0.5 \mathrm{~cm}, X_{A_{2}}=0.6 \mathrm{~cm}\right)$ и $H_{A}=0.25 \mathrm{~cm}$ $\left(H_{A_{1}}=0.425 \mathrm{~cm}, \quad X_{A_{2}}=0.675 \mathrm{~cm}\right)$. Выбор высоты $H_{A}=0.1 \mathrm{~cm}$ мотивирован наличием экспериментальных данных по визуальному наблюдению структуры пеннинговского разряда при давлениях порядка $p \sim 10^{-3}[15]$ и $p \sim 10^{-5}$ Torr [16]. К сожалению, наблюдений структуры разряда Пеннинга при исследованных в настоящей работе давлений автору неизвестны. Тем не менее именно малая высота анода позволяет в перспективе выполнить такие эксперименты. Для некоторых вариантов проведено
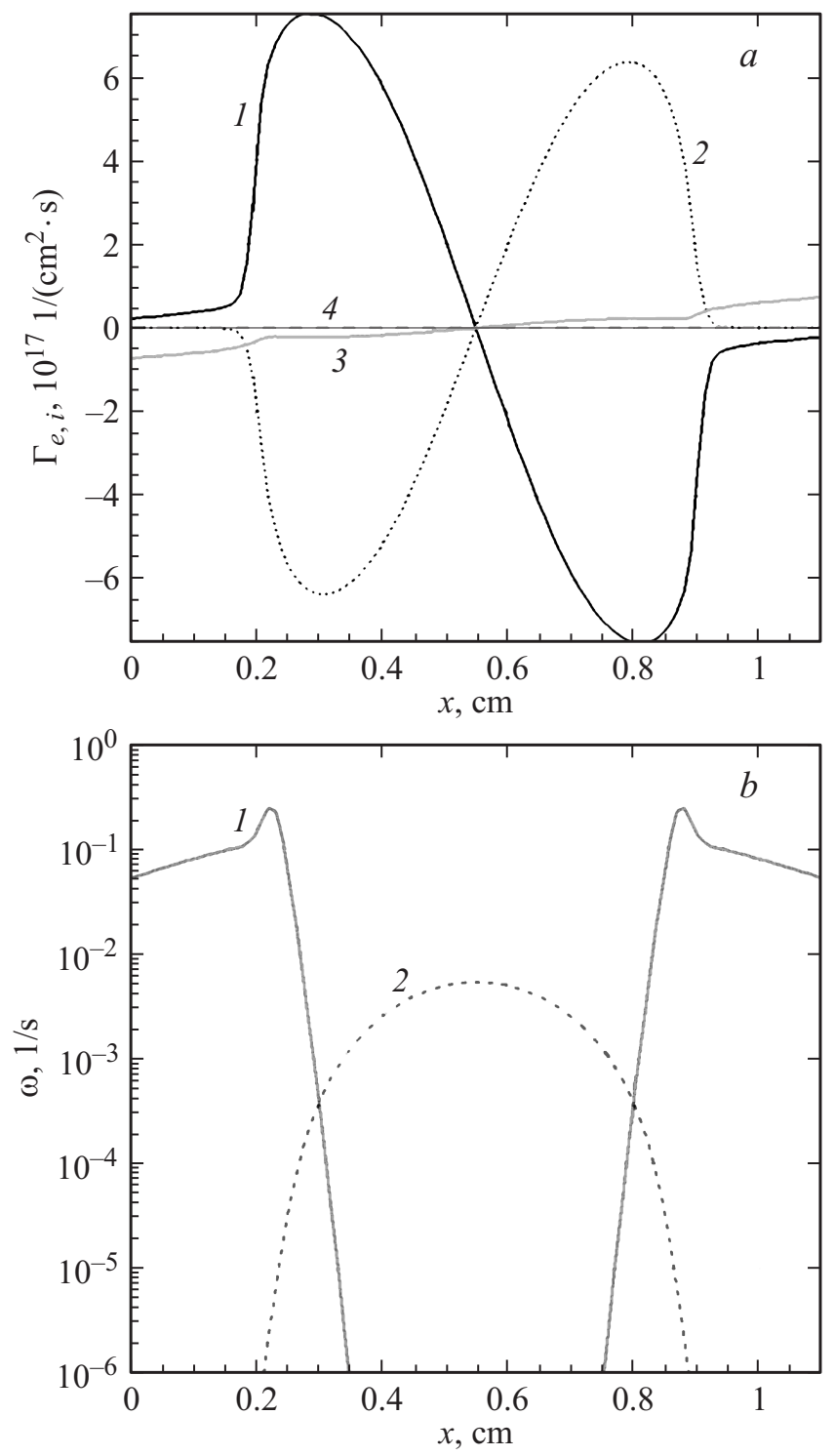

Рис. 5. Осевые распределения параметров разряда вдоль оси симметрии пеннинговского разряда с анодом высотой $H_{A}=0.1 \mathrm{~cm}$ при $p=1$ Torr, $B_{x}=0.1 \mathrm{~T}, \varepsilon=200 \mathrm{~V} ; a-$ плотность дрейфовых (сплошные кривые) и диффузионных (штрихи) потоков электронов $(1,2)$ и ионов $(3,4) ; b-$ скорость ионизации (1) и рекомбинации (2).

также исследование предельно низких напряжений, при которых возможно существование разряда Пеннинга.

Сначала изучим структуру тлеющего разряда с кольцевым анодом высотой $H_{A}=0.1 \mathrm{~cm}$. Распределения концентраций электронов и ионов в разрядном промежутке при $p=1$ Torr, $\varepsilon=100-300 \mathrm{~V}$ показаны на рис. 2 . При $\varepsilon=100 \mathrm{~V}$ разряд без магнитного поля погасает. При включении магнитного поля в центральной приосевой области образуется облако квазинейтральной плазмы (рис. 2,a). С увеличением ЭДС в диапазоне $\varepsilon=100-300 \mathrm{~V}$ происходит увеличение концентраций в области квазинейтральной плазмы от $n_{e} \approx n_{i}=6.8 \cdot 10^{9}$ 

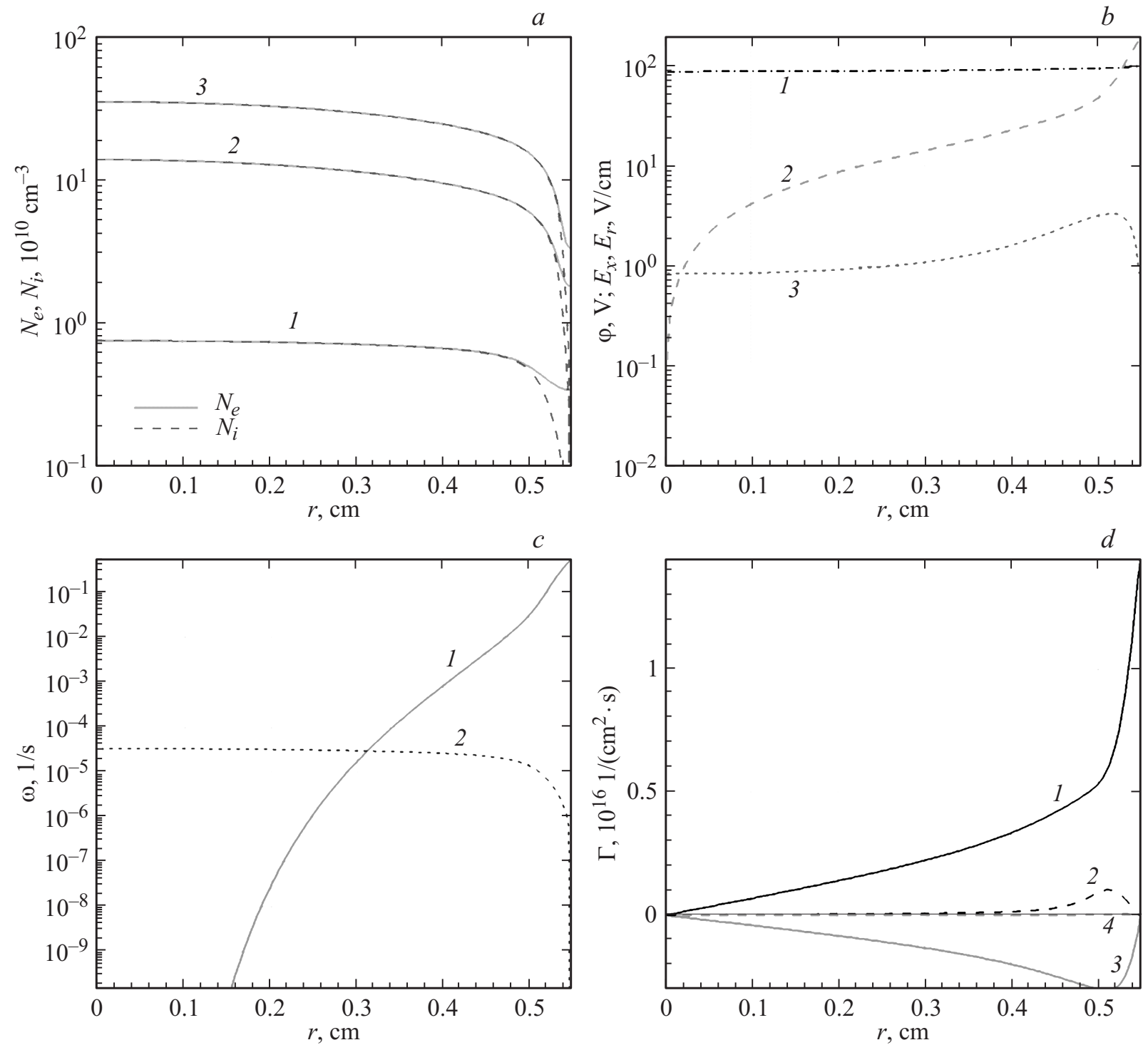

Рис. 6. Радиальные распределения параметров разряда в центральном сечении $(x=0.55 \mathrm{~cm})$ анода высотой $H_{A}=0.1 \mathrm{~cm}$ при $p=1$ Torr, $B_{x}=0.1 \mathrm{~T} ; a-$ концентрация электронов (сплошные кривые) и ионов (штриховые кривые) при $\varepsilon=100(1), 200(2)$ и $300 \mathrm{~V}(3) ; b$ - электрический потенциал (1), осевая (2) и радиальная (3) компоненты электрического поля в центральном сечении; $c$ - скорость ионизации $(1)$ и рекомбинации $(2)$ при $\varepsilon=100 \mathrm{~V} ; d-$ плотности потоков электронов $(1,2)$ и ионов $(3,4)$ за счет дрейфа $(1,3)$ и диффузии $(2,4)$ при $\varepsilon=100 \mathrm{~V}$.

(рис. $2, a)$ до $3.1 \cdot 10^{11} \mathrm{~cm}^{3}$ (рис. 2,c). При этом размеры плазменного облака в осевом направлении заметно возрастают.

На рис. 3, где показаны осевые распределения концентраций заряженных частиц, хорошо идентифицируются указанная область квазинейтральной плазмы и области пространственного заряда вблизи катода и антикатода. Концентрация электронов в непосредственной близости от катодных электродов определяется вторичной ионэлектронной эмиссией. Осевые распределения электрического потенциала и напряженности электрического поля $E_{x}$ для трех ЭДС $\varepsilon=100,200,300 \mathrm{~V}$ показаны на рис. $3, b$. В приосевой области разряда электрический потенциал достигает величины $\varphi=86,165,226 \mathrm{~V}$ соот- ветственно. Заметим, что при $\varepsilon=300 \mathrm{~V}$ напряженность электрического поля на катоде и антикатоде достигает значений $\sim 1700 \mathrm{~V} / \mathrm{cm}$, что уже несколько превышает достоверный диапазон, для которого справедлива формула Таунсенда (15) с аппроксимационными коэффициентами (16).

Осевые распределения усредненных скоростей электронов и ионов, приведенные на рис. 4, соответствуют показанным распределениям полей. Ускоряемые в катодном слое ионы до скоростей $\left|v_{i, x}\right| \sim 5.0 \cdot 10^{6}$, $1.5 \cdot 10^{7}, 2.25 \cdot 10^{7} \mathrm{~cm} / \mathrm{s}$ (соответственно при $\varepsilon=100$, 200,300 V) при подходе к катоду и антикатоду вызывают ион-электронную эмиссию. Усредненные осевые скорости электронов у катода составляют соответственно 

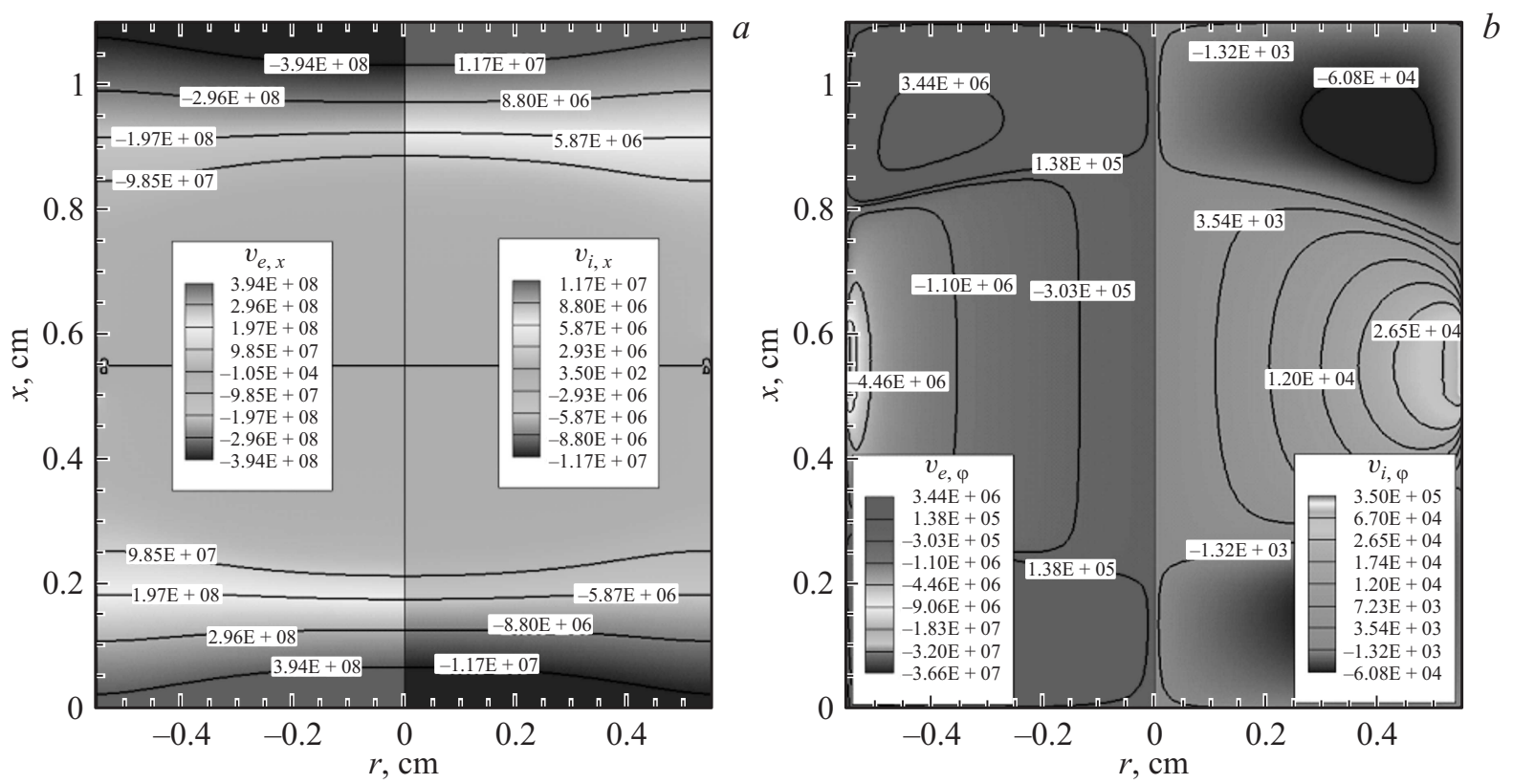

Рис. 7. Поля продольных $v_{e, x}, v_{i, x}(a)$ и азимутальных $v_{e, \varphi}, v_{i, \varphi}(b)$ скоростей движения электронов и ионов (в ст/s) при $p=1$ Torr, $B_{x}=0.1 \mathrm{~T}, \varepsilon=200 \mathrm{~V}$.

$\left|v_{e, x}\right| \sim 1.7 \cdot 10^{8}, 4.9 \cdot 10^{8}, 7.6 \cdot 10^{8} \mathrm{~cm} / \mathrm{s}$. Напомним, что в рамках диффузионно-дрейфовой модели усредненная скорость определяется по локальным параметрам разряда (4) и имеет две составляющие: дрейфовую и диффузионную. Из рис. 5, $a$ видно, что у катода основная составляющая потока ионов и электронов - дрейфовая. Заметная диффузионная составляющая потока электронов формируется на границе катодных слоев и области квазинейтральной плазмы (эта граница хорошо идентифицируется по осевому распределению электронов и ионов на рис. 3, $a$ и осевых распределений напряженности электрического поля (рис. $3, b$ ). Подчеркнем, что в соответствии с (4) и (5) наибольшие значения плотностей потоков заряженных частиц определяются не только их скоростью, но и концентрацией. Именно поэтому наибольшая величина осевой плотности потока электронов достигается вблизи границы катодных слоев. Поскольку плотность потока электронов во многом определяет источниковое слагаемое $\omega$ в правой части (1) и (2), то максимальная величина скорости ионизации также достигается в этой области (рис. $5, b$ ). В области квазинейтральной плазмы превалирует рекомбинация ионов и электронов.

Распределения параметров разряда по нормали к оси симметрии в центральном сечении анода показаны на рис. 6. На рис. 6, $a$ даны радиальные распределения концентраций ионов и электронов при ЭДС $\varepsilon=100$, 200,300 V. Квазинейтральная плазма занимает большую часть объема внутри анода. Анодный слой имеет малую толщину, около $0.05 \mathrm{~cm}$. Радиальное распределение электрического потенциала, осевой и радиальной компонент напряженности электрического поля приведены на рис. $6, b$. Как было видно из рис. $3, b$ величина $E_{x}$ на оси в рассматриваемом сечении близка к нулю. Из рис. $6, b$ следует, что вблизи анода $E_{x}>200 \mathrm{~V} / \mathrm{cm}$. Максимум радиальной компоненты электрического поля $E_{r}$ достигает здесь небольшой величины $\sim 3 \mathrm{~V} / \mathrm{cm}$. Отсюда следует, что основной вклад в ионизацию вблизи анода вносят электроны, дрейфующие в продольном, а не в радиальном направлении.

Радиальные распределения источниковых компонент в правой части (1) и (2) показаны на рис. 6, $c$. В приосевом направлении превалирует рекомбинация. В анодном слое и в квазинейтральной плазме при $r>0.3 \mathrm{~cm}$ ионизационные процессы превосходят рекомбинационные. Из рис. $6, d$ видно, что основной компонентой радиальной плотности потока электронов и ионов является дрейфовая. Диффузионные составляющие потоков электронов и ионов малы. Абсолютные величины плотностей потоков в радиальном направлении на 1-2 порядка ниже, чем в осевом направлении.

Поля продольных $v_{e, x}, v_{i, x}$ и азимутальных $v_{e, \varphi}, v_{i, \varphi}$ скоростей движения электронов и ионов показаны на рис. 7. Наибольшие осевые скорости электронов и ионов наблюдаются в приосевых областях. Их абсолютные значения различаются почти на 2 порядка. Наибольшие скорости ионов при подлете к катоду составляют $\sim 1.2 \cdot 10^{7} \mathrm{~cm} / \mathrm{s}$.

Важным элементом структуры пеннинговского разряда является усредненное азимутальное движение электронов и ионов. Наибольшая скорость азимутального движения электронов наблюдается у поверхности анода и достигает $v_{e, \varphi} \sim 3.7 \cdot 10^{7} \mathrm{~cm} / \mathrm{s}$. Ионы со средней азимутальной скоростью $v_{i, \varphi} \sim 2.7 \cdot 10^{4} \mathrm{~cm} / \mathrm{s}$ вращаются 

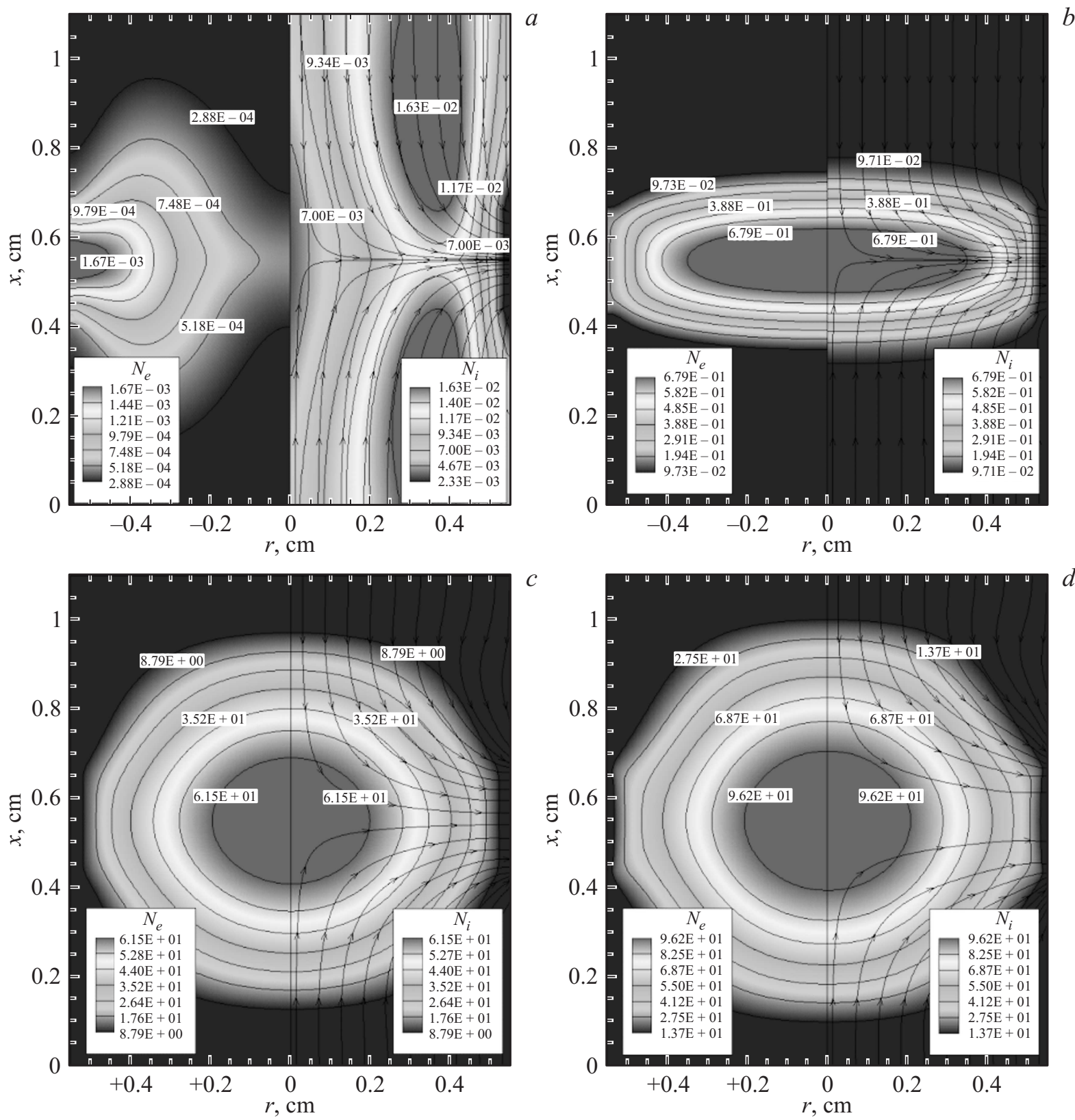

Рис. 8. Концентрация электронов (слева) и ионов (справа) в пеннинговском разряде с высотой анода $H_{A}=0.25 \mathrm{~cm}$ при $p=1$ Тоrr: $a-\varepsilon=100 \mathrm{~V}, B_{x}=0 ; b-\varepsilon=100 \mathrm{~V}, B_{x}=0.1 \mathrm{~T} ; c-\varepsilon=200 \mathrm{~V}, B_{x}=0.1 \mathrm{~T} ; d-\varepsilon=300 \mathrm{~V}, B_{x}=0.1 \mathrm{~T}$.

в противоположном направлении. Примечательно, что в исследуемой области наблюдаются две подобласти с противоположным направлением вращения электронов и ионов. В рассматриваемом случае одна такая подобласть располагается у анода, а другая - у катода. Как следует из (4) и (6), направление вращения непосредственно связано с направлением радиальных потоков заряженных частиц. Вблизи анода поток электронов направлен к аноду, а вблизи катода имеется небольшая составляющая, направленная к оси симметрии. Для ионов наблюдается обратная ситуация.

Отмеченные выше закономерности для кольцевого анода являются типичными для пеннинговского разряда при давлении порядка 1 Torr. Тем не менее при увеличении высоты анода наблюдаются некоторые изменения в его структуре. На рис. 8 показаны поля концентраций ионов и электронов для анода высотой $H_{A}=0.25 \mathrm{~cm}$ при $p=1$ Torr в разряде без магнитного поля при $\varepsilon=100 \mathrm{~V}$, a также при $B_{x}=0.1 \mathrm{~T}$ для трех значений ЭДС в диапазоне $\varepsilon=100-300 \mathrm{~V}$.

При отсутствии магнитного поля наибольшая концентрация электронов наблюдается вблизи поверхности анода, а ионов - вблизи катода. Квазинейтральной плазмы в разрядной области нет. Заметим, что $\varepsilon=100 \mathrm{~V}$ обеспечивает существование разряда практически вблизи нижнего предела своего существования. Увеличение $\varepsilon$ 
приводит к более полному заполнению газоразрядного промежутка плазмой. По мере увеличения ЭДС разряд все более и более приобретает сферическую форму (см. последовательно рис. $8, b-d)$.

Осевые распределения концентраций электронов и ионов, а также продольной компоненты электрического поля показаны на рис. 9, $a, b$, позволяют установить основные закономерности их изменения при вариации давления и индукции магнитного поля.

При отсутствии магнитного поля осевые распределения заряженных частиц демонстрируют отсутствие области квазинейтральности (кривая $1, p=1$ Torr, $\varepsilon=100 \mathrm{~V})$. При включении магнитного поля с индук-
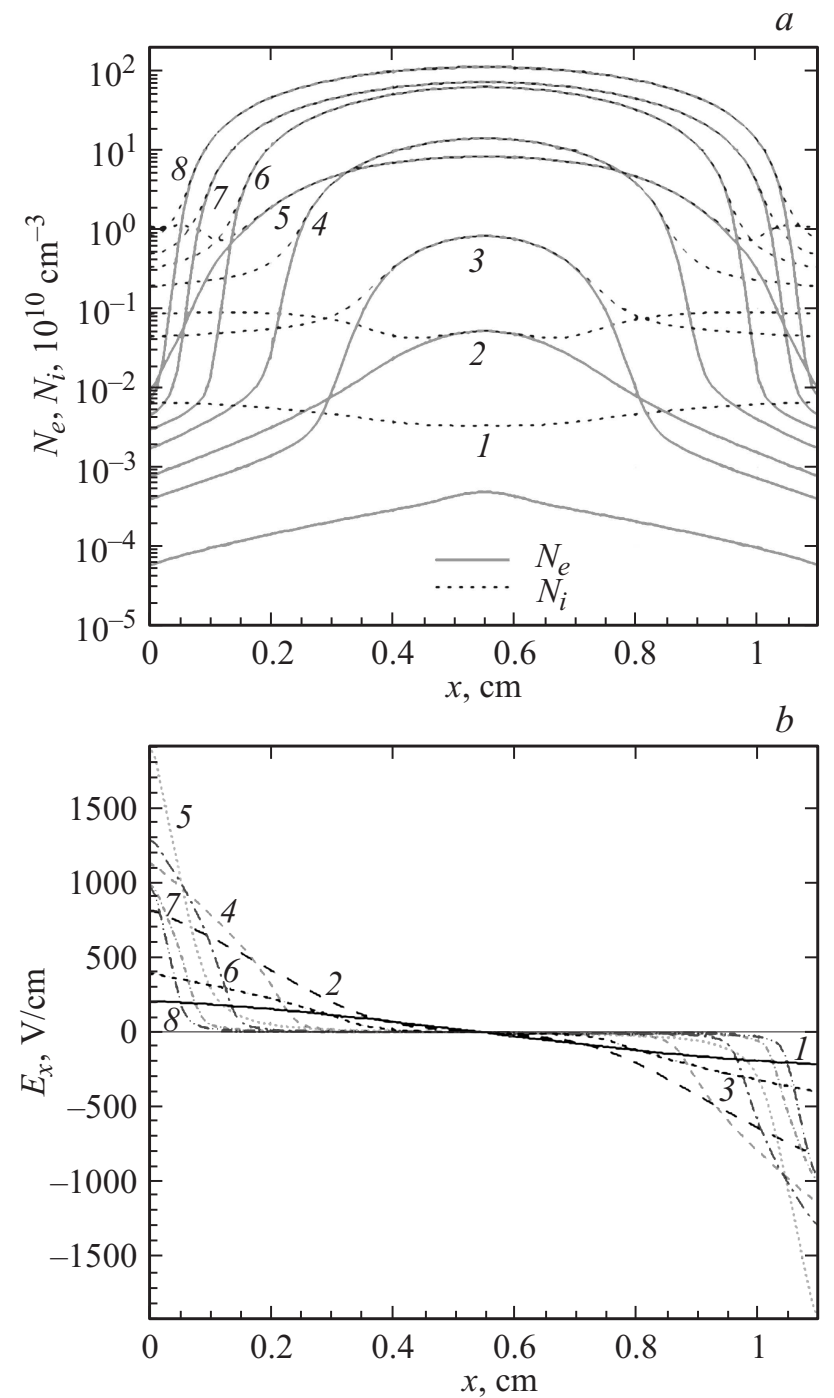

Рис. 9. Распределения концентраций электронов (сплошные кривые) и ионов (штриховые) $(a)$, а также напряженности электрического поля вдоль оси симметрии пеннинговского разряда с высотой анода $H_{A}=0.25 \mathrm{~cm}(b): 1-p=1$ Torr, $\varepsilon=100 \mathrm{~V}, B_{x}=0,2-p=1$ Torr, $\varepsilon=100 \mathrm{~V}, B_{x}=0.1 \mathrm{~T}$, $3-p=1$ Torr, $\varepsilon=200 \mathrm{~V}, B_{x}=0,4-p=1$ Torr, $\varepsilon=200 \mathrm{~V}$, $B_{x}=0.1 \mathrm{~T}, 5-p=5$ Torr, $\varepsilon=200 \mathrm{~V}, B_{x}=0.1 \mathrm{~T}, 6-$ $p=0.5$ Torr, $\varepsilon=200 \mathrm{~V}, B_{x}=0.1 \mathrm{~T}, 7-p=1$ Torr, $\varepsilon=200 \mathrm{~V}$, $B_{x}=0.3 \mathrm{~T}, 8-p=1$ Torr, $\varepsilon=200 \mathrm{~V}, B_{x}=0.6 \mathrm{~T}$.

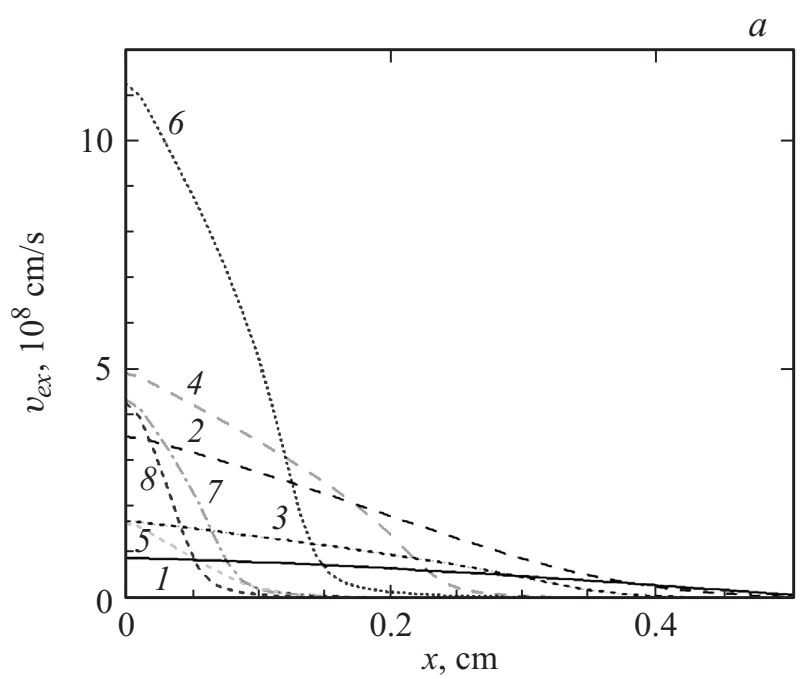

$b$

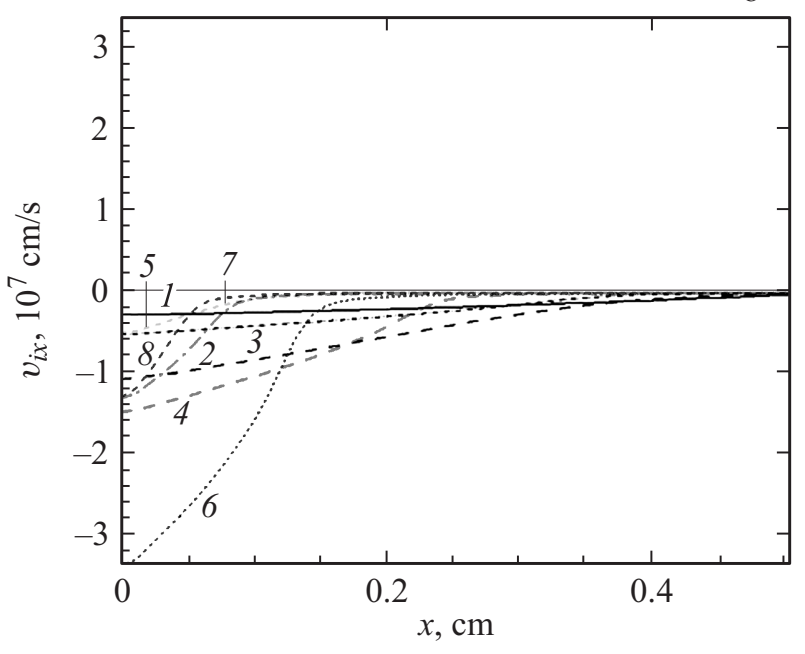

Рис. 10. Распределения осевой скорости электронов (a) и ионов $(b)$ вдоль оси симметрии пеннинговского разряда с высотой анода $H_{A}=0.25 \mathrm{~cm}: 1-p=1$ Torr, $\varepsilon=100 \mathrm{~V}$, $B_{x}=0,2-p=1$ Torr, $\varepsilon=100 \mathrm{~V}, B_{x}=0.1 \mathrm{~T}, 3-p=1$ Torr, $\varepsilon=200 \mathrm{~V}, B_{x}=0,4-p=1$ Torr, $\varepsilon=200 \mathrm{~V}, B_{x}=0.1 \mathrm{~T}$, $5-p=5$ Torr, $\varepsilon=200 \mathrm{~V}, B_{x}=0.1 \mathrm{~T}, 6-p=0.5$ Torr, $\varepsilon=200 \mathrm{~V}, B_{x}=0.1 \mathrm{~T}, 7-p=1 \mathrm{Torr}, \varepsilon=200 \mathrm{~V}, B_{x}=0.3 \mathrm{~T}$, $8-p=1$ Torr, $\varepsilon=200 \mathrm{~V}, B_{x}=0.6 \mathrm{~T}$.

цией $B_{x}=0.1 \mathrm{~T}$ приводят к заметному возрастанию концентраций ионов и электронов (кривая 2).

Увеличение ЭДС до $\varepsilon=200 \mathrm{~V}$ приводит к заметному возрастанию концентраций электронов и ионов как без магнитного поля (кривая 3), так и при $B_{x}=0.1 \mathrm{~T}$ (кривая 4).

Если при $\varepsilon=200 \mathrm{~V}$ повысить давление до $p=5$ Torr, то концентрация в центре анода изменяется незначительно, но возрастают осевые размеры разряда (кривая 5). Если же давление в газе уменьшить до $p=0.5$ Torr, то наблюдается и увеличение концентраций заряженных частиц, и более полное заполнение плазмой газоразрядного промежутка (кривая 6). Важно, что увеличение индукции магнитного поля до $B_{x}=0.6 \mathrm{~T}$ при 

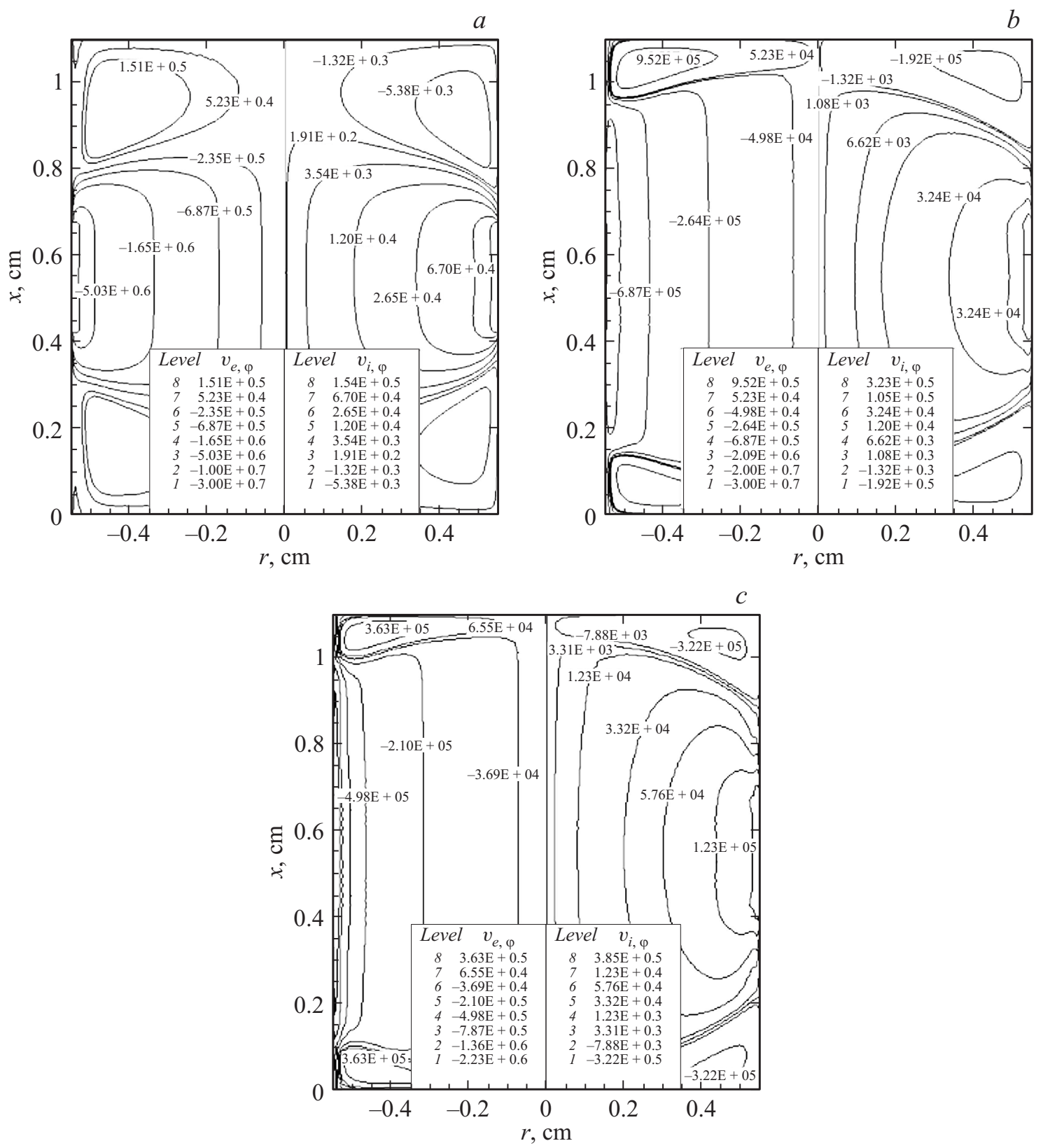

Рис. 11. Азимутальные скорости электронов (слева) и ионов (справа) при $p=1$ Torr, $\varepsilon=100 \mathrm{~V}, B_{x}=0.1(a), 0.3(b), 0.6 \mathrm{~T}(c)$.

$p=1$ Torr оказывается более эффективным для увеличения концентрации заряженных частиц, чем уменьшение давления (кривые 7 и 8 ).

На рис. 9, $b$ показано осевое распределение электрического поля $E_{x}$ при разных давлениях, ЭДС и индукциях магнитного поля. Наименьшее значение $E_{x}$ наблюдается при $\varepsilon=100 \mathrm{~V}, p=1$ Torr, $B_{x}=0$ (кривая 1 ). Увеличение $\varepsilon$ до $200 \mathrm{~V}$ без магнитного поля приводит к росту напряженности электрического поля вблизи катода в два раза (кривая 3). Включение магнитного поля с индукцией $B_{x}=0.1 \mathrm{~T}$ также увеличивает $E_{x}$ у катода (сравните кривые 1 и 2). Наибольшей величины $E_{x}$ достигает при увеличении давления до $p=5$ Torr (кривая 5). При уменьшении давления до $p=0.5$ Torr рост поля у катода также оказывается заметным (кривая 6). При этом все большая часть разрядного промежутка становится квазинейтральной, а значит области с относительно высокими электрическими полями смещаются ближе к катоду и антикатоду.

К возрастанию электрического поля вблизи катода приводит увеличение индукции магнитного поля до $B_{x}=0.3$ и $0.6 \mathrm{~T}$ (соответственно кривые 7 и 8 ).

Распределения напряженности электрического поля $E_{x}$ вдоль оси симметрии объясняют зависимости осевых составляющих скоростей ионов и электронов от давления, ЭДС и индукции магнитного поля. На рис. 10 показаны распределения скоростей электронов $v_{e, x}$ и ионов $v_{i, x}$ при тех же исходных данных, при которых 
Воль-амперные характеристики разряда Пеннинга

\begin{tabular}{c|c|c|c|c}
\hline$\varepsilon, \mathrm{V}$ & $B_{x}, \mathrm{~T}$ & $p$, Torr & $I, \mathrm{~A}$ & $V, \mathrm{~V}$ \\
\hline \multicolumn{5}{c}{$H_{A}=0.1 \mathrm{~cm}$} \\
\hline 100 & 0 & 1 & $0.133 \cdot 10^{-3}$ & 99 \\
\hline 100 & 0.1 & 1 & $0.378 \cdot 10^{-3}$ & 97 \\
\hline 200 & 0.1 & 1 & $0.411 \cdot 10^{-2}$ & 175 \\
\hline 300 & 0.1 & 1 & $0.105 \cdot 10^{-1}$ & 237 \\
\hline \multicolumn{5}{|c|}{$H_{A}=0.25 \mathrm{~cm}$} \\
\hline 100 & 0 & 1 & $0.829 \cdot 10^{-4}$ & 99 \\
\hline 100 & 0.1 & 1 & $0.408 \cdot 10^{-3}$ & 97 \\
\hline 100 & 0.3 & 1 & $0.493 \cdot 10^{-2}$ & 70 \\
\hline 100 & 0.6 & 1 & $0.719 \cdot 10^{-2}$ & 57 \\
\hline 200 & 0 & 1 & $0.259 \cdot 10^{-2}$ & 184 \\
\hline 200 & 0.1 & 1 & $0.423 \cdot 10^{-2}$ & 175 \\
\hline 300 & 0.1 & 1 & $0.107 \cdot 10^{-1}$ & 236 \\
\hline 300 & 0 & 5 & $0.231 \cdot 10^{-1}$ & 161 \\
\hline 300 & 0.1 & 5 & $0.239 \cdot 10^{-1}$ & 157 \\
& & & & \\
\hline
\end{tabular}

анализировались распределения концентраций ионов и электронов, а также напряженностей электрического поля. Показаны распределения скоростей вблизи катода. Вблизи антикатода распределения такие же, с точностью до знака. Из рис. 10 видно, что наибольшую скорость ионы и электроны достигают при наименьшем давлении (кривые 6). При $p=1$ Torr и $\varepsilon=200 \mathrm{~V}$ увеличение индукции в диапазоне $B_{x}=0.1-0.6 \mathrm{~T}$ (кривые $4,7,8$ ) приводит к уменьшению осевых скоростей. В магнитном поле $B_{x}=0.1 \mathrm{~T}$ увеличение давления до $p=5$ Torr уменьшает наибольшие осевые скорости (кривые 5 ), а уменьшение давления до $p=0.5$ Torr увеличивает скорости (кривые 6). При $B_{x}=0.1 \mathrm{~T}$ и давлении $p=1$ Torr увеличение ЭДС приводит к росту абсолютных величин осевых составляющих скоростей электронов и ионов (кривые 2 и 4 ).

В заключение отметим основные закономерности изменения осредненной азимутальной скорости вращения электронов и ионов. На рис. 11 показаны азимутальные скорости ионов и электронов в пеннинговском разряде при $p=1$ Torr, ЭДС $\varepsilon=100 \mathrm{~V}, H_{A}=0.25 \mathrm{~cm}$ при последовательном увеличении индукции магнитного поля. Главной особенностью является увеличение осевых размеров области однонаправленного вращения ионов и электронов. И, наоборот, область противоположного их вращения у катода и антикатода уменьшается.

В таблице приведены вольт-амперные характеристики разряда Пеннинга, полученные в расчетах по диффузионно-дрейфовой модели. Изменение высоты анода практически не изменяет ток через разряд и паде- ние напряжения на разрядном промежутке. Включение магнитного поля несколько снижает падение напряжения, но заметно увеличивает ток через разряд $I$. Увеличение ЭДС приводит к возрастанию падения напряжения и полного тока через разряд.

\section{Заключение}

Разработанная диффузионно-дрейфовая модель пеннинговского разряда для давлений $p \geq 1$ Torr позволила получить более полные качественное и количественное представления об эволюции его пространственной структуры в зависимости от таких характеристик разряда, как размеров анода, ЭДС в диапазоне $\varepsilon=100-200 \mathrm{~V}$, давления в диапазоне $p=0.5-5$ Torr и индукции осевого магнитного поля в диапазоне $B_{x}=0.1-0.6$ Т. Исследована геометрия разряда Пеннинга, типичная для газонаполненных нейтронных трубок.

Численным моделированием подтвержден установленный ранее в теоретических работах факт усредненного вращения электронов и ионов в противоположных направлениях. Показано, что в разрядном промежутке возможно существование, как минимум, двух областей разнонаправленного движения ионов и электронов. Получены азимутальные скорости такого вращения, которые составляют для электронов $v_{e, \varphi} \sim 10^{7} \mathrm{~cm} / \mathrm{s}$ и для ионов $v_{i, \varphi} \sim 10^{5} \mathrm{~cm} / \mathrm{s}$.

Исследованы закономерности в распределениях осевых скоростей ионов и электронов при уменьшении давления, увеличения ЭДС и индукции магнитного поля. Показано, что наибольшие скорости осевого движения электронов и ионов соответственно $v_{e, x} \sim 10^{9} \mathrm{~cm} / \mathrm{s}$ и $v_{i, x} \sim 3.0 \cdot 10^{7} \mathrm{~cm} / \mathrm{s}$ достигаются при наименьших из исследованных давлений, $p=0.5$ Torr. При фиксированном давлении $p=1$ Torr ускорение движения заряженных частиц достигается при увеличении ЭДС. Рост индукции магнитного поля замедляет скорость усредненного аксиального движения электронов и ионов.

Дальнейшее снижение давления в рамках данной модели не является оправданным, поскольку изменяется определяющий механизм упругих и ионизирующих столкновений.

Работа поддержана Российским научным фондом (грант № 16-11-10275)

\section{Список литературы}

[1] Penning F.M. // Fhysica III. 1936. N 9. P. 873-894.

[2] Грановский В.Л. Электрический ток в газе. Установившийся ток. М.: Наука. 1971. 543 с.

[3] Марков В.Г., Прохорович Д.Е., Садилин А.Г., Щитов Н.Н. // Успехи прикладной физики. 2013. Т. 1. № 1. C. 23-29.

[4] Габович М.Д., Плешивцев Н.В., Семашко Н.Н. Пучки ионов и атомов для управляемого термоядерного синтеза и технологических целей. М.: Энергоатомиздат. 1986. $249 \mathrm{c}$. 
[5] Ситников М.М. // ЖТФ. 1938. Т. 8. Вып. 17. С. 1527-1547.

[6] Мухамедов Р.Ф. // ЖТФ. 1973. Т. 43. Вып. 8. С. 1677-1684.

[7] Hirsch E.N. // Brit. J. Appl. Phys. 1964. Vol. 15. P. 1535-1544.

[8] Коротаев Ю.В., Мешков И.Н., Поляков В.Н., Смирнов А.В., Сыресин Е.М., Лей З., Транквилль Ж. // ЖТФ. 1997. Т. 67. Вып. 11. С. 124-126.

[9] Никулин С.П. // ЖТФ. 1998. Т. 68. Вып. 7. С. 56-63.

[10] Зюлькова Л.А., Козырев А.В., Проскуровский Д.И. // ЖТФ. 2005. Т. 75. Вып. 11. С. 59-64.

[11] Браун C. Элементарные процессы в плазме газового разряда. М.: Гос. Изд-во литературы в области атомной науки и техники, 1961. 323 c. (Brown S.C. Basic Data of Plasma Physics. Technology Press of M.I.T. and Wiley, 1966).

[12] Суржиков С.T. // Письма в ЖТФ. 2017. Т. 43. Вып. 3. C. 64-71.

[13] Surzhikov S.T., Shang J.S. // Plasma Sources Sci. Technol. 2014. Vol. 23. P. 054017.

[14] Surzhikov S.T. Computational Physics of Electric Discharges in Gas Flows. Berlin/Boston: Walter de Gruyter GmbH. 2013. $428 \mathrm{p}$.

[15] Крейндель Ю.Е., Ионов А.С. // ЖТФ. 1964. Т. 34. Вып. 7. C. 1199-1205.

[16] Рейхрудель Э.М., Чернетский А.В., Михневич В.В., Васильева И.А. // ЖТФ. 1952. Т. 22. Вып. 12. С. 1945-1966. 